\begin{tabular}{|c|c|}
\hline Title: & $\begin{array}{l}\text { Analysis of Current Harmonics in Inverter-Driven Interior Permanent Magnet } \\
\text { Synchronous Machines }\end{array}$ \\
\hline Authors: & $\begin{array}{l}\text { Gemassmer, Tobias } \\
\text { Braun, Michael }\end{array}$ \\
\hline Institute: & $\begin{array}{l}\text { Karlsruhe Institute of Technology (KIT) } \\
\text { Elektrotechnisches Institut (ETI) } \\
\text { Electrical Drives and Power Electronics }\end{array}$ \\
\hline Type: & Conference Proceedings \\
\hline Published at: & $\begin{array}{l}\text { 15th European Conference on Power Electronics and Applications (EPE), 2013, Lille, } \\
\text { France, Sept. 2-6, } 2013 \\
\text { Publisher: IEEE } \\
\text { Year: } 2013 \\
\text { ISBN: } 978-1-4799-0115-9 \\
\text { Pages: } 5333-5342\end{array}$ \\
\hline Hyperlinks: & DOI: $10.1109 / E P E .2013 .6634724$ \\
\hline
\end{tabular}

(c) 2013 IEEE. Personal use of this material is permitted. Permission from IEEE must be obtained for all other uses, in any current or future media, including reprinting/republishing this material for advertising or promotional purposes, creating new collective works, for resale or redistribution to servers or lists, or reuse of any copyrighted component of this work in other works. 


\title{
Analysis of Current Harmonics in Inverter-Driven Interior Permanent Magnet Synchronous Machines
}

\author{
Tobias Gemaßmer and Michael Braun \\ Karlsruhe Institute of Technology (KIT) \\ Elektrotechnisches Institut (ETI) - Electrical Drives and Power Electronics \\ Kaiserstr. 12, 76131 Karlsruhe, Germany \\ Phone: +49 (0) $721608-42476$ \\ Fax: +49 (0) 721-358854 \\ Email: tobias.gemassmer@kit.edu \\ URL: http://www.eti.kit.edu
}

\section{Keywords}

$<<$ Permanent magnet motor $>>,<<$ Converter machine interactions $>>,<<$ Harmonics $>>,<<$ Modulation strategy $>>$

\begin{abstract}
This paper presents the analysis of current harmonics in an inverter-driven interior permanent magnet machine for different modulation methods. In contrast to other approaches, the influence of the saliency $\left(L_{\mathrm{d}} \neq L_{\mathrm{q}}\right)$ on the harmonics is being considered. By use of the rms-value of the currents, analytically closed equations can be deduced and tedious Fourier analyses of single harmonics can be avoided.

The derivation of the analytic equations is described and the results are verified by numerical simulation as well as by experimental results.
\end{abstract}

\section{Introduction}

The influence of the modulation method on the current waveform in converter systems with high pulse rate has been investigated in different publications (e.g. [1] - [4]). There the calculation of the current harmonics has been done with a three-phase symmetric load at the converter output, which is represented by three equal inductances (fig. 1). However, in the case of magnetic anisotropic permanent magnet synchronous machines the inductance in each of the three output phases differs from the others and variates depending on the rotor position $\gamma$ of the machine.

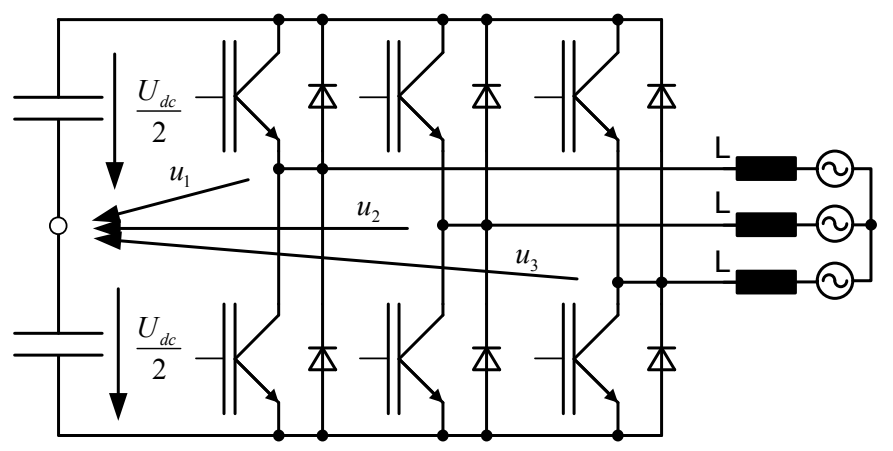

Figure 1: Voltage source inverter with symmetric load

Additionally, the phase angle between the stator voltage of an interior permanent magnet synchronous machine (IPMSM) and the rotor position $\gamma$ depends on the stator current, which makes the values of the inductances at the inverter output also dependent on the operation point of the machine. 
This paper presents the estimation of the current harmonics in a drive system consisting of a voltage source inverter (VSI, fig. 1) and an IPMSM depending on the modulation depth and the phase angle $\varphi_{U}$ of the stator voltage for different modulation methods. Verification of the developed formulas is performed by numerical simulation of a drive system. Measurements on a machine test bench have also been accomplished in order to validate the derived formulas.

\section{Calculation of current harmonics}

In order to estimate the current harmonics, first the machine model and the switching states of the inverter are being described. Thereon, the current waveform within one half pulse period of the inverter will be examined. Subsequently, the current harmonics related to one full period of the inverter output voltages $u_{1}, u_{2}, u_{3}$ will be evaluated.

\section{Machine model}

Since the inductances of the IPMSM are constant in the rotor oriented dq-reference frame (fig. 2a), this frame will be used for the estimation of the current waveform. Thus the IPMSM can be described by the stator voltage equations and the equations for the flux linkages:

$$
\begin{array}{ll}
u_{\mathrm{d}}=R_{\mathrm{S}} \cdot i_{\mathrm{d}}+\frac{\mathrm{d} \psi_{\mathrm{d}}}{\mathrm{d} t}-\omega \cdot \psi_{\mathrm{q}} & u_{\mathrm{q}}=R_{\mathrm{s}} \cdot i_{\mathrm{q}}+\frac{\mathrm{d} \psi_{\mathrm{q}}}{\mathrm{d} t}+\omega \cdot \psi_{\mathrm{d}} \\
\psi_{\mathrm{d}}=L_{\mathrm{d}} \cdot i_{\mathrm{d}}+\psi_{\mathrm{PM}} & \psi_{\mathrm{q}}=L_{\mathrm{q}} \cdot i_{\mathrm{q}}
\end{array}
$$

The functions for the current component waveforms can be obtained by solving eq. (1) and (2) for the current derivatives. Assuming the inductances $L_{\mathrm{d}}$ and $L_{\mathrm{q}}$ and the speed $\omega$ as constant and neglecting the stator resistance $R_{\mathrm{S}}$, this results in a system of ordinary differential equations:

$$
\frac{\mathrm{d} i_{\mathrm{d}}}{\mathrm{d} t}=\frac{u_{\mathrm{d}}+\omega \cdot L_{\mathrm{q}} \cdot i_{\mathrm{q}}(t)}{L_{\mathrm{d}}} \quad \frac{\mathrm{d} i_{\mathrm{q}}}{\mathrm{d} t}=\frac{u_{\mathrm{q}}-\omega \cdot\left(L_{\mathrm{d}} \cdot i_{\mathrm{d}}(t)+\psi_{\mathrm{PM}}\right)}{L_{\mathrm{q}}}
$$

Solving this system of ordinary differential equations, the functions for the current components become

$$
\begin{aligned}
& i_{\mathrm{d}}(t)=\frac{\left[\left(L_{\mathrm{d}} \cdot i_{\mathrm{d}, 0}+\psi_{\mathrm{PM}}\right) \cdot \omega-u_{\mathrm{q}}\right] \cdot \cos (\omega t)}{\omega \cdot L_{\mathrm{d}}}+\frac{\left(\omega \cdot L_{\mathrm{q}} \cdot i_{\mathrm{q}, 0}+u_{\mathrm{d}}\right) \sin (\omega t)+u_{\mathrm{q}}-\omega \cdot \psi_{\mathrm{PM}}}{\omega \cdot L_{\mathrm{d}}} \\
& i_{\mathrm{q}}(t)=\frac{\left[-\left(L_{\mathrm{d}} \cdot i_{\mathrm{d}, 0}+\psi_{\mathrm{PM}}\right) \cdot \omega+u_{\mathrm{q}}\right] \cdot \sin (\omega t)}{\omega \cdot L_{\mathrm{q}}}+\frac{\left(u_{\mathrm{d}}+\omega \cdot L_{\mathrm{q}} \cdot i_{\mathrm{q}, 0}\right) \cdot \cos (\omega t)-u_{\mathrm{d}}}{\omega \cdot L_{\mathrm{q}}}
\end{aligned}
$$

with the initial values $i_{\mathrm{d}}(t=0)=i_{\mathrm{d}, 0}$ and $i_{\mathrm{q}}(t=0)=i_{\mathrm{q}, 0}$.

In case of a high pulse rate, the variation of the rotor position angle $\Delta \gamma=\Delta \omega t$ during one half pulse period can also be neglected. Therefore the small-angle approximation $\sin (\omega t) \approx \omega t$ and $\cos (\omega t) \approx 1$ can be used. This simplifies eq. (4) and (5) to

$$
i_{\mathrm{d}}(t)=\frac{u_{\mathrm{d}}+\omega \cdot L_{\mathrm{q}} \cdot i_{\mathrm{q}, 0}}{L_{\mathrm{d}}} \cdot t+i_{\mathrm{d}, 0} \quad i_{\mathrm{q}}(t)=\frac{u_{\mathrm{q}}-\omega \cdot\left(L_{\mathrm{d}} \cdot i_{\mathrm{d}, 0}+\psi_{\mathrm{PM}}\right)}{L_{\mathrm{q}}} \cdot t+i_{\mathrm{q}, 0}
$$

The voltage components $u_{\mathrm{d}}$ and $u_{\mathrm{q}}$ in eq. (6) depend on the discrete switching states of the inverter.

\section{Switching states of the inverter}

The converter voltage space vector is defined as

$$
\underline{u}_{\mathrm{s}}=\frac{2}{3} \cdot\left(u_{1}+\underline{a} \cdot u_{2}+\underline{a}^{2} \cdot u_{3}\right) \quad \text { with } \quad \underline{a}=-\frac{1}{2}+j \cdot \frac{\sqrt{3}}{2}
$$

Assuming the location of the desired voltage space vector in sector I in fig. $2 \mathrm{~b}$, the discrete converter voltage space vectors used during the pulse period are

$$
\underline{u}_{1}=\frac{2}{3} \cdot U_{\mathrm{dc}} \quad \underline{u}_{2}=\frac{2}{3} \cdot U_{\mathrm{dc}} \cdot\left(\frac{1}{2}+j \frac{\sqrt{3}}{2}\right)
$$




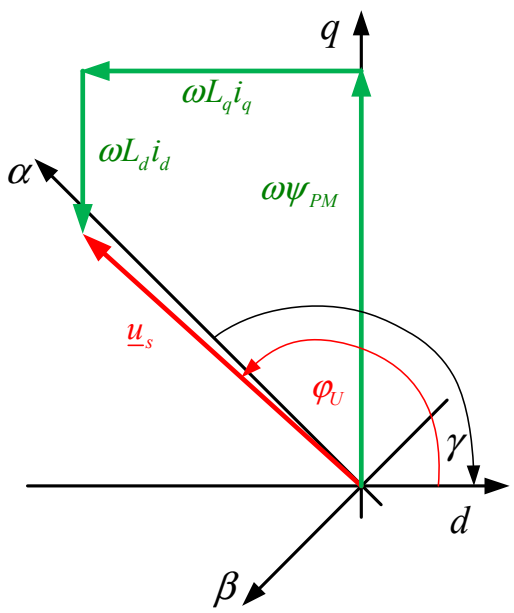

(a)

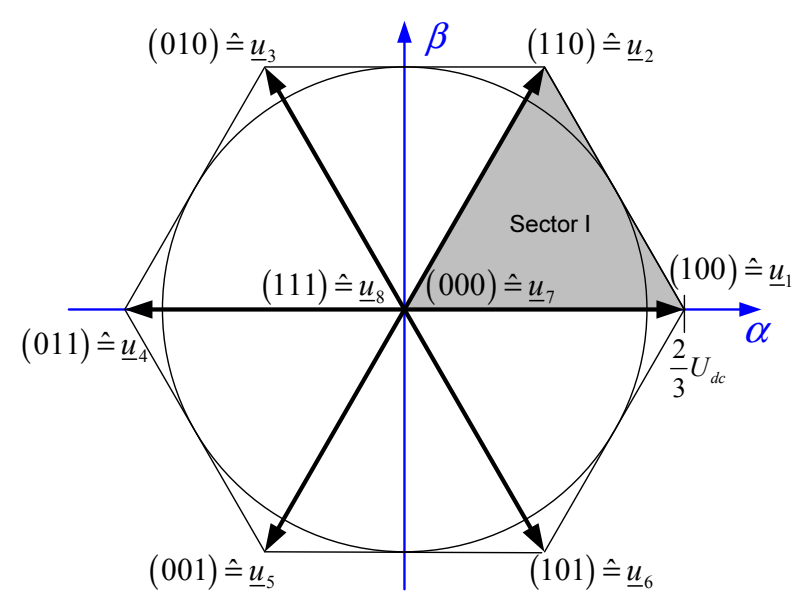

(b)

Figure 2: (a): Stator voltage space vector in the rotary dq-frame and its location in the stationary $\alpha \beta$-frame. (b): Switching states and possible output voltage of the inverter.

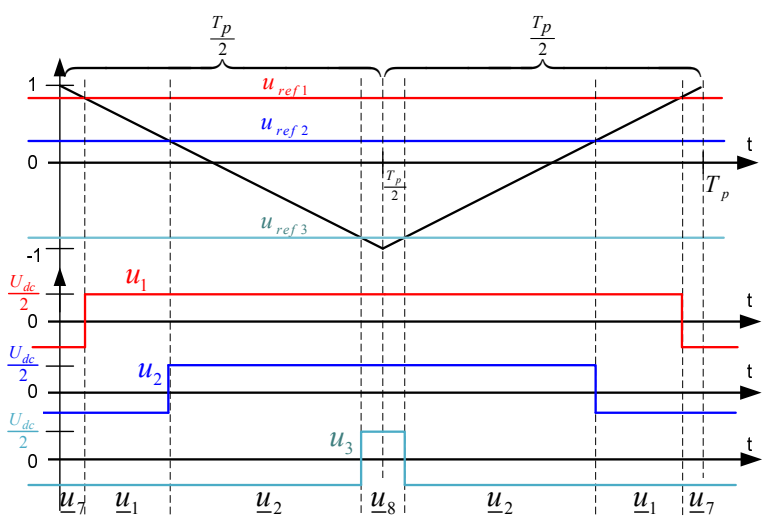

(a)

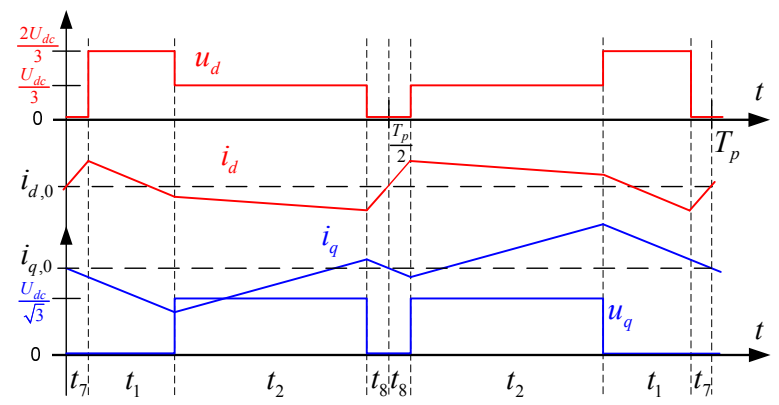

(b)

Figure 3: Exemplary switching state sequence in sector I (a) and exemplary current waveform at $\gamma=0$ (b).

Additionally, one or two of the freewheeling states 7 and 8 can be inserted in order to achieve the desired mean value of the voltage space vector during the pulse period $T_{\mathrm{p}}$. For sinusoidal phase modulation, the switching state sequence in sector I is shown in fig. $3 \mathrm{a}$.

The effect on the current components in the dq-frame can be obtained by transforming the discrete voltage space vectors from the stationary to the rotating frame. The resulting voltage components are

$$
\begin{aligned}
& u_{\mathrm{d}, 1}=\mathfrak{R}\left\{\underline{u}_{\alpha \beta, 1} \cdot e^{-j \cdot \gamma}\right\}=\frac{2}{3} \cdot U_{\mathrm{dc}} \cdot \cos \gamma \\
& u_{\mathrm{d}, 2}=\mathfrak{R}\left\{\underline{u}_{\alpha \beta, 2} \cdot e^{-j \cdot \gamma}\right\}=\frac{1}{3} \cdot U_{\mathrm{dc}} \cdot(\cos \gamma+\sqrt{3} \cdot \sin \gamma) \\
& u_{\mathrm{d}, 7}=u_{\mathrm{d}, 8}=0 \\
& u_{\mathrm{q}, 1}=\mathfrak{I}\left\{\underline{u}_{\alpha \beta, 1} \cdot e^{-j \cdot \gamma}\right\}=-\frac{2}{3} \cdot U_{\mathrm{dc}} \cdot \sin \gamma \\
& u_{\mathrm{q}, 2}=\mathfrak{I}\left\{\underline{u}_{\alpha \beta, 2} \cdot e^{-j \cdot \gamma}\right\}=\frac{1}{3} \cdot U_{\mathrm{dc}} \cdot(\sqrt{3} \cdot \cos \gamma-\sin \gamma) \\
& u_{\mathrm{q}, 7}=u_{\mathrm{q}, 8}=0
\end{aligned}
$$

Fig. $3 \mathrm{~b}$ shows exemplary waveforms of $i_{\mathrm{d}}(t)$ and $i_{\mathrm{q}}(t)$ within one pulse period assuming $\gamma=0$. 


\section{Current harmonics during one half pulse period}

During the following considerations, the mean value of the inverter output voltage within one half pulse period will be assumed to be equal to the stator voltage of the machine. The inner voltage components

$$
e_{\mathrm{d}}=-\omega \cdot L_{\mathrm{q}} \cdot i_{\mathrm{q}, 0} \quad e_{\mathrm{q}}=\omega \cdot\left(L_{\mathrm{d}} \cdot i_{\mathrm{d}, 0}+\psi_{\mathrm{PM}}\right)
$$

are approximately constant during $\frac{T_{\mathrm{p}}}{2}$ for high pulse rates [1]. They can be expressed by use of the modulation depth $M$, which is defined as (cf. [2])

$$
M=\frac{2 \cdot\left|\underline{u}_{\mathrm{s}}\right|}{U_{\mathrm{dc}}}
$$

For steady-state operation the stator voltage is equal to the inner voltage. The angle $\varphi_{U}$ of the stator voltage space vector in the dq-frame (fig. 2a) then depends on the inner voltage components:

$$
\left|\underline{u}_{\mathrm{s}}\right|=\sqrt{e_{\mathrm{d}}^{2}+e_{\mathrm{q}}^{2}} \quad \varphi_{\mathrm{U}}=\arctan \frac{e_{\mathrm{q}}}{e_{\mathrm{d}}}
$$

The components of the inner voltage can be expressed in terms of the modulation depth by

$$
e_{\mathrm{d}}=\frac{1}{2} \cdot M \cdot U_{\mathrm{dc}} \cdot \cos \varphi_{\mathrm{U}} \quad e_{\mathrm{q}}=\frac{1}{2} \cdot M \cdot U_{\mathrm{dc}} \cdot \sin \varphi_{\mathrm{U}}
$$

The current during each of the switching states $x \in\{1,2,7,8\}$ in one half pulse period then is

$$
i_{\mathrm{d}}(t)=\frac{u_{\mathrm{d}, \mathrm{x}}-\frac{1}{2} \cdot M \cdot U_{\mathrm{dc}} \cdot \cos \varphi_{\mathrm{U}}}{L_{\mathrm{d}}} \cdot t+i_{\mathrm{d}, \mathrm{k}-1} \quad i_{\mathrm{q}}(t)=\frac{u_{\mathrm{q}, \mathrm{x}}-\frac{1}{2} \cdot M \cdot U_{\mathrm{dc}} \cdot \sin \varphi_{\mathrm{U}}}{L_{\mathrm{q}}} \cdot t+i_{\mathrm{q}, \mathrm{k}-1}
$$

with $i_{\mathrm{d} / \mathrm{q}, \mathrm{k}-1}$ being the value of $i_{\mathrm{d} / \mathrm{q}}$ at the moment of changing the switching state. To calculate the current harmonics, the mean values of $i_{\mathrm{d}}$ and $i_{\mathrm{q}}$ during one half pulse period are not of interest. Therefore the initial current component values $i_{\mathrm{d}, 0}$ and $i_{\mathrm{q}, 0}$ at $t=0$ can be set to zero.

To get the square of the rms-value of the harmonic current during one half pulse period, the squares of the two currents are integrated to:

$$
\Delta i_{\mathrm{s}}^{2}=\frac{2}{T_{\mathrm{p}}} \int_{0}^{\frac{T_{\mathrm{p}}}{2}} \frac{i_{\mathrm{d}}^{2}(t)+i_{\mathrm{q}}^{2}(t)}{2} \mathrm{~d} t
$$

Since the voltage space vector applied to the machine needs to be equal to the stator voltage of the machine $\underline{u}_{\mathrm{s}}=e_{\mathrm{d}}+j e_{\mathrm{q}}$, the turn-on times of the different switching states for sinusoidal pulse width modulation (SPWM) can be estimated directly from the stator voltage (cf. fig. 3a):

$$
\begin{aligned}
\frac{t_{7}}{T_{\mathrm{p}} / 2} & =\frac{1}{2} \cdot\left[1-M \cdot \cos \left(\varphi_{\mathrm{U}}+\gamma\right)\right] \\
\frac{t_{1}}{T_{\mathrm{p}} / 2} & =\frac{1}{2} \cdot\left[1-M \cdot \cos \left(\varphi_{\mathrm{U}}+\gamma-\frac{2 \pi}{3}\right)\right]-\frac{t_{7}}{T_{\mathrm{p}} / 2} \\
\frac{t_{2}}{T_{\mathrm{p}} / 2} & =\frac{1}{2} \cdot\left[1-M \cdot \cos \left(\varphi_{\mathrm{U}}+\gamma-\frac{4 \pi}{3}\right)\right]-\frac{t_{7}}{T_{\mathrm{p}} / 2}-\frac{t_{1}}{T_{\mathrm{p}} / 2} \\
\frac{t_{8}}{T_{\mathrm{p}} / 2} & =1-\frac{t_{7}}{T_{\mathrm{p}} / 2}-\frac{t_{1}}{T_{\mathrm{p}} / 2}-\frac{t_{2}}{T_{\mathrm{p}} / 2}
\end{aligned}
$$

Eq. (21) to (24) are only valid for sector I of the voltage plane in fig. $2 \mathrm{~b}$, so the rotor position angle has to be set to the corresponding interval

$$
\gamma \in\left\{-\varphi_{U} \ldots-\varphi_{U}+\frac{\pi}{3}\right\}
$$

With the estimation of the turn-on times of the switching states, the square of the harmonic current rms value $\Delta i_{\mathrm{s}}^{2}$ within one half pulse period is determined. 


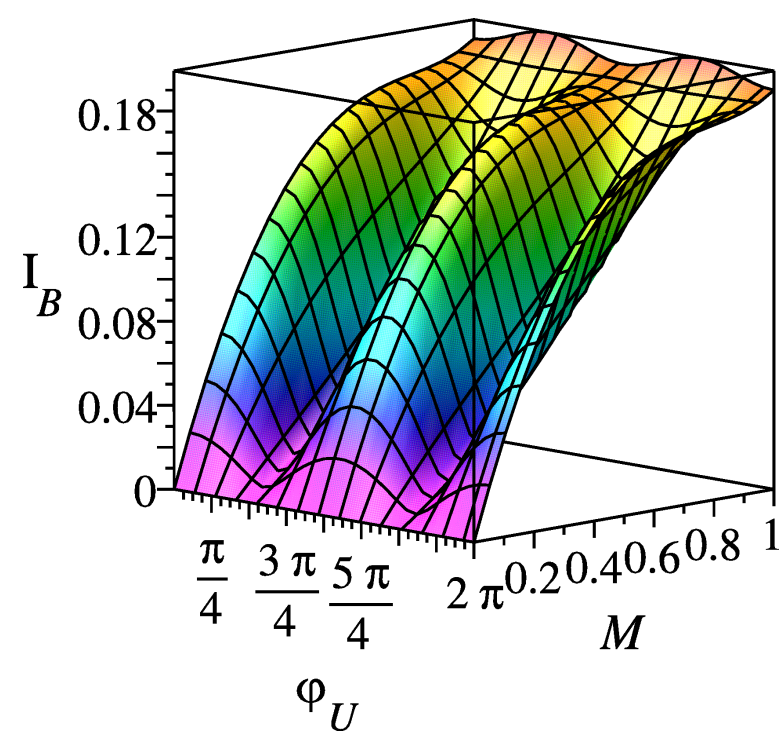

(a)

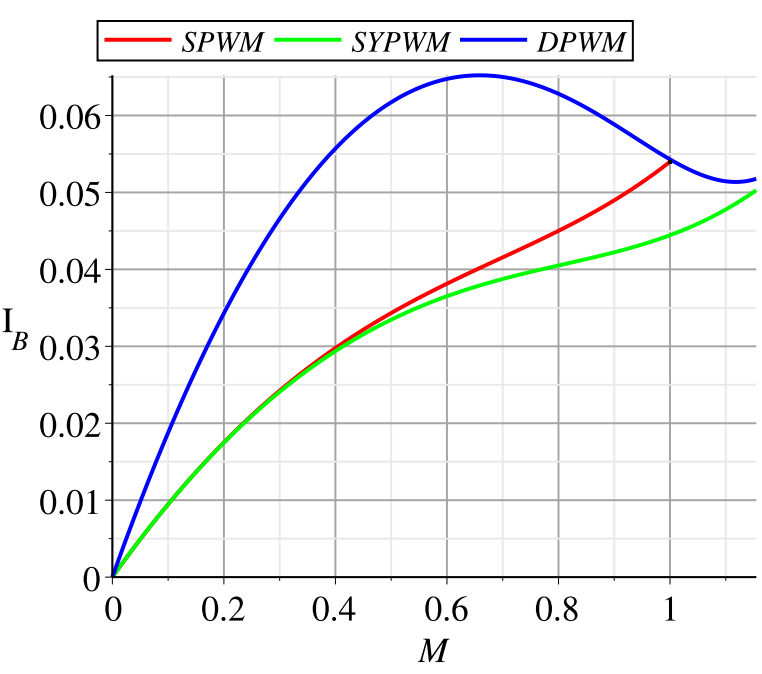

(b)

Figure 4: (a): Current harmonics for SPWM. (b): Current harmonics for magnetic isotropic machines for use of different modulation methods

\section{RMS value of harmonic currents related to one full period of the fundamental}

Calculation of the mean value of the particular harmonic currents related to the pulse periods within sector I leads to the square of the resulting harmonic current rms value:

$$
\Delta I^{2}=\frac{1}{m} \cdot \sum_{\mathrm{m}} \Delta i_{\mathrm{s}}^{2}
$$

Due to the symmetries of the output characteristics of the inverter, this is equivalent to the mean value for an entire fundamental [2]. Assuming high pulse rate in relation to the fundamental, eq. (26) can be approximated by integration:

$$
\Delta I^{2} \approx \frac{3}{\pi} \cdot \int_{-\varphi_{U}}^{-\varphi_{U}+\pi / 3} \Delta i_{\mathrm{s}}^{2} \mathrm{~d} \gamma
$$

Evaluating $\Delta I^{2}$ leads to the result for sinusoidal modulation (SPWM):

$$
\begin{aligned}
\Delta I_{\mathrm{SPWM}}^{2} & =\left(\frac{T_{\mathrm{P}}}{2}\right)^{2} \cdot\left(\frac{U_{\mathrm{dc}}}{l L_{\mathrm{d}}}\right)^{2} \cdot M^{2} \cdot\left[\frac{1}{128}\left(1+\left(l^{2}-1\right)\left(\cos \left(\varphi_{\mathrm{U}}\right)\right)^{2}\right) \cdot M^{2}-\right. \\
& \left.\frac{\sqrt{3}\left(12\left(\cos \left(\varphi_{\mathrm{U}}\right)\right)^{2}\left(l^{2}-1\right)+11-l^{2}\right)}{360 \pi} \cdot M+\frac{1}{96}\left(1+\left(l^{2}-1\right)\left(\cos \left(\varphi_{\mathrm{U}}\right)\right)^{2}\right)\right]
\end{aligned}
$$

with the saliency factor

$$
l=\frac{L_{\mathrm{q}}}{L_{\mathrm{d}}}
$$

The result for $I_{\mathrm{B}}=\Delta I_{\mathrm{SPWM}} /\left(\frac{T_{\mathrm{P}}}{2} \cdot \frac{U_{\mathrm{dc}}}{l L_{\mathrm{d}}}\right)$ for a machine with $L_{\mathrm{d}}=0.3 \mathrm{mH}$ and $L_{\mathrm{q}}=1.5 \mathrm{mH}$ is shown in fig. 4a. In eq. (28) as well as in fig. 4a the dependency of $\Delta I$ on the angle $\varphi_{U}$ of the stator voltage can clearly be seen. The minimum of the current harmonics for constant modulation depth appears at $\varphi_{U}=\frac{\pi}{2}$ and $\varphi_{\mathrm{U}}=\frac{3 \pi}{2}$, which corresponds to the direction of $L_{\mathrm{q}}$. The highest value of the current harmonics occur at $\varphi_{U}=0$ and $\varphi_{U}=\pi$, as the voltage space vector is located in the direction of the smaller inductance $L_{\mathrm{d}}$. However, at modulation depths near $M \approx 1$ the current harmonics become higher for $\varphi_{\mathrm{U}}=\frac{\pi}{2}$ and $\varphi_{\mathrm{U}}=\frac{3 \pi}{2}$ than for $\varphi_{\mathrm{U}}=0$ and $\varphi_{\mathrm{U}}=\pi$. 


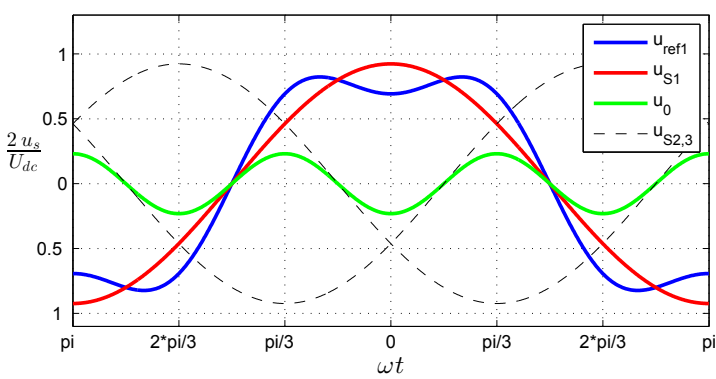

(a)

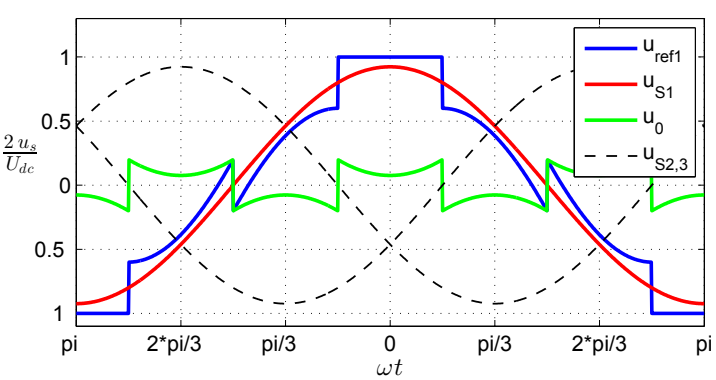

(c)

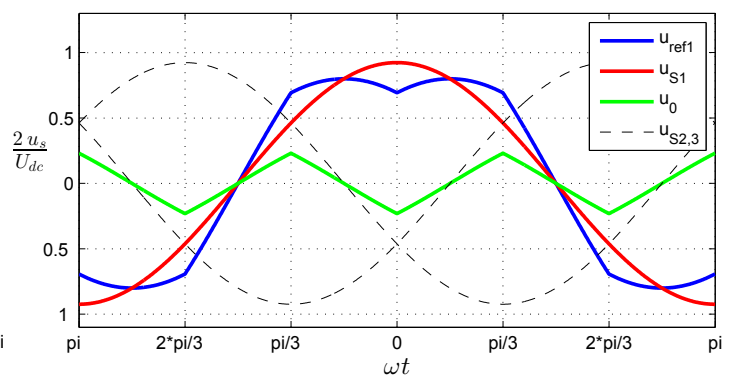

(b)

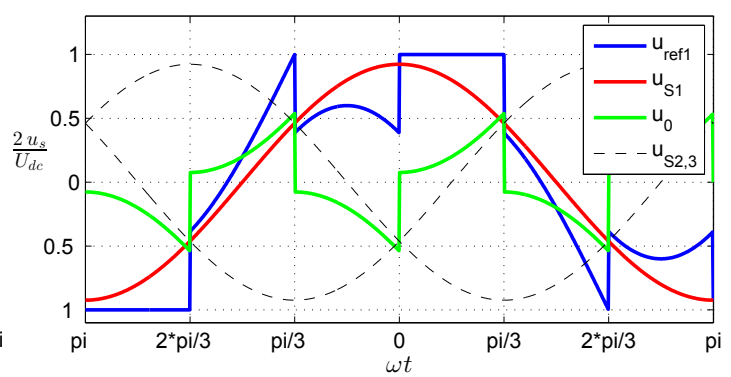

(d)

Figure 5: Reference functions. (a): 3rdPWM. (b): SYPWM. (c): DPWM1. (d): DPWM2.

In case of a magnetic isotropic PMSM, we have $L_{\mathrm{d}}=L_{\mathrm{q}}$ and therefore $l=1$. Eq. (28) then simplifies to

$$
\Delta I_{\text {SPWM }}^{2}=\left(\frac{T_{\mathrm{P}}}{2}\right)^{2} \cdot\left(\frac{U_{\mathrm{dc}}}{L_{\mathrm{d}}}\right)^{2} \cdot M^{2} \cdot\left(\frac{1}{128} \cdot M^{2}-\frac{\sqrt{3}}{36 \pi} \cdot M+\frac{1}{96}\right)
$$

This corresponds to the red line in fig. 4b. There is no dependency of the current harmonics on the angle $\varphi_{\mathrm{U}}$ for machines with $L_{\mathrm{d}}=L_{\mathrm{q}}$. Fig. $4 \mathrm{~b}$ also shows the values of the current harmonics for other modulation methods, which will be discussed in the next section. In case of $L_{\mathrm{d}}=L_{\mathrm{q}}$ they do not depend on the angle $\varphi_{\mathrm{U}}$ either.

\section{Utilization of different modulation methods}

The described method of estimating current harmonics can easily be applied to different modulation methods. As the duration of the two active switching states $t_{2}$ and $t_{3}$ needs to be the same for each modulation method, only the turn-on times of the freewheeling states need to be adjusted.

For magnetic isotropic machines, the optimal modulation method in order to reduce the harmonic current rms value is realized by adding a third harmonic (3rdPWM) with an amplitude of $\frac{M_{3}}{M_{1}}=\frac{1}{4}$ [2]. The resulting waveforms of the reference functions can be seen in fig. $5 \mathrm{a}$.

The turn-on times of the freewheeling-states for this modulation method result to

$$
\begin{aligned}
& \frac{t_{7,3 \mathrm{rdPWM}}}{T_{\mathrm{p}} / 2}=\frac{1}{2}\left[1-M \cdot \cos \left(\varphi_{\mathrm{U}}+\gamma\right)+\frac{M}{4} \cdot \cos \left(3 \cdot \varphi_{\mathrm{U}}+\gamma\right)\right] \\
& \frac{t_{8,3 \mathrm{rdPWM}}}{T_{\mathrm{p}} / 2}=1-\frac{t_{7,3 \mathrm{rdPWM}}}{T_{\mathrm{p}} / 2}-\frac{t_{1}}{T_{\mathrm{p}} / 2}-\frac{t_{2}}{T_{\mathrm{p}} / 2}
\end{aligned}
$$

Applying these turn-on times to eq. (20) and solving eq. (27) yields the solution for 3rdPWM:

$$
\begin{aligned}
\Delta I_{3 \mathrm{rdPWM}}^{2} & =\left(\frac{T_{\mathrm{P}}}{2}\right)^{2} \cdot\left(\frac{U_{\mathrm{dc}}}{l L_{\mathrm{d}}}\right)^{2} \cdot M^{2} \cdot\left[\frac{7}{1024} \frac{\left(1+\left(l^{2}-1\right)\left(\cos \left(\varphi_{\mathrm{U}}\right)\right)^{2}\right)\left(-\frac{3 \sqrt{3}}{4}+\pi\right)}{\pi} \cdot M^{2}-\right. \\
& \left.\frac{\sqrt{3}\left(12\left(\cos \left(\varphi_{\mathrm{U}}\right)\right)^{2}\left(l^{2}-1\right)+11-l^{2}\right)}{360 \pi} \cdot M+\frac{1}{96}\left(1+\left(l^{2}-1\right)\left(\cos \left(\varphi_{\mathrm{U}}\right)\right)^{2}\right)\right]
\end{aligned}
$$




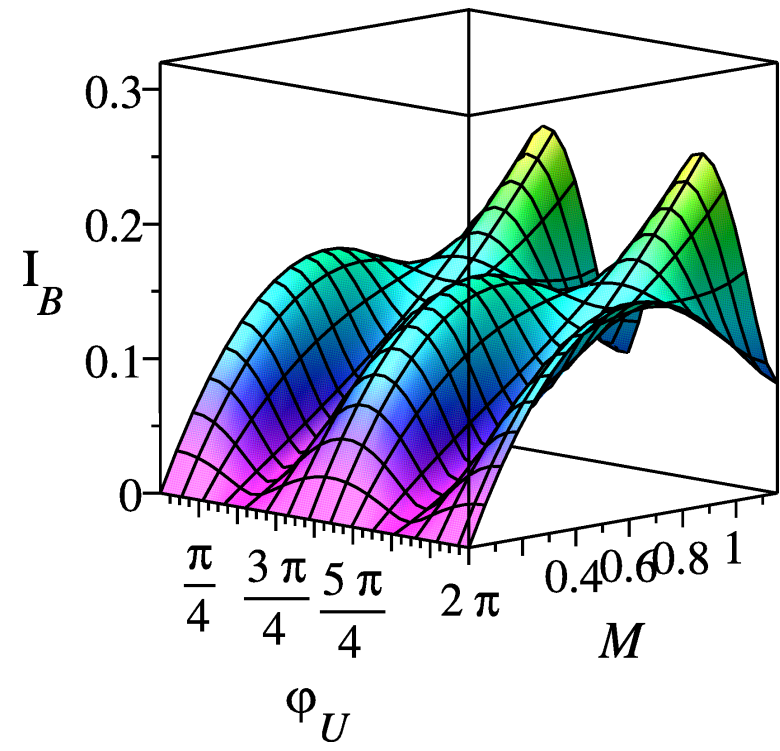

(a)

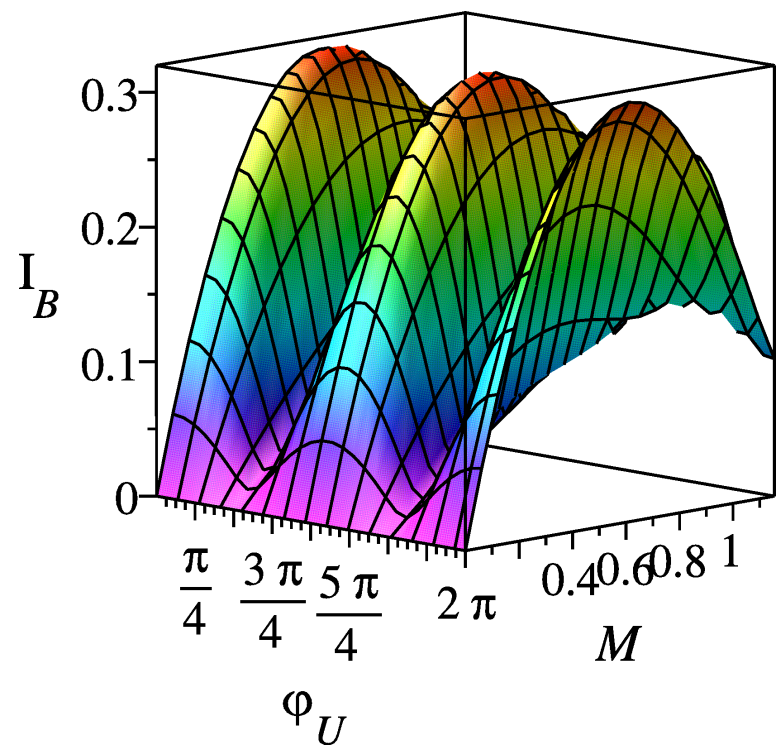

(b)

Figure 6: Current harmonics $I_{\mathrm{B}}=\Delta I /\left(\frac{T_{\mathrm{P}}}{2} \frac{U_{\mathrm{dc}}}{l L_{\mathrm{q}}}\right)$ for SYPWM (a) and DPWM2 (b).

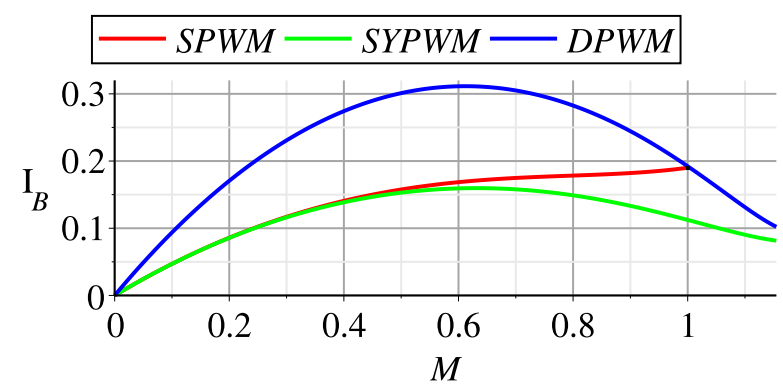

(a)

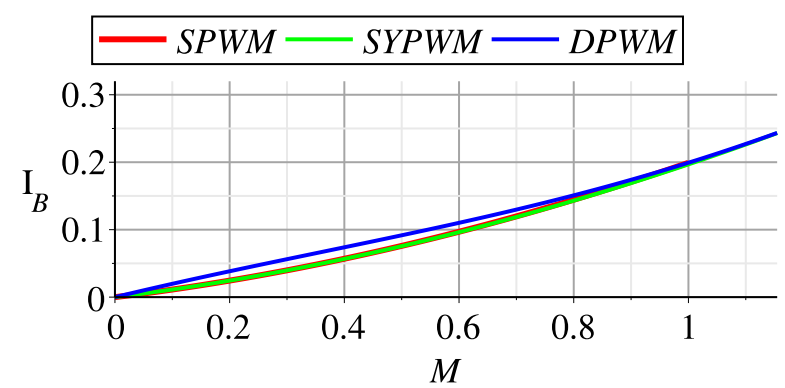

(b)

Figure 7: Current harmonics $I_{\mathrm{B}}=\Delta I /\left(\frac{T_{\mathrm{P}}}{2} \frac{U_{\mathrm{dc}}}{l L_{\mathrm{q}}}\right)$ for use of different modulation methods. (a): $\varphi_{\mathrm{U}}=\pi$. (b): $\varphi_{\mathrm{U}}=\frac{\pi}{2}$.

In case of distributing the remaining time $\frac{T_{\mathrm{p}}}{2}-t_{1}-t_{2}$ symmetrical at the beginning and at the end of the half pulse period (SYPWM, fig. 5b), which represents a suboptimal approximation for 3rdPWM [2], the duration of the two freewheeling-states resolves to

$$
\frac{t_{7, \mathrm{SYPWM}}}{T_{\mathrm{p}} / 2}=\frac{t_{8, \mathrm{SYPWM}}}{T_{\mathrm{p}} / 2}=\frac{1}{2} \cdot\left(1-\frac{t_{1}}{T_{\mathrm{p}} / 2}-\frac{t_{2}}{T_{\mathrm{p}} / 2}\right)
$$

Applying the turn-on times in eq. (34) to $\Delta i_{\mathrm{S}}$ leads to the result for SYPWM:

$$
\begin{aligned}
& \Delta I_{\text {SYPWM }}^{2}=\left(\frac{T_{\mathrm{P}}}{2}\right)^{2} \cdot\left(\frac{U_{\mathrm{dc}}}{l L_{\mathrm{d}}}\right)^{2} \cdot M^{2} \cdot\left[\frac{3}{256} \frac{\left(1+\left(l^{2}-1\right)\left(\cos \left(\varphi_{\mathrm{U}}\right)\right)^{2}\right)\left(-\frac{3 \sqrt{3}}{4}+\pi\right)}{\pi} \cdot M^{2}-\right. \\
& \left.\frac{\sqrt{3}\left(12\left(\cos \left(\varphi_{\mathrm{U}}\right)\right)^{2}\left(l^{2}-1\right)+11-l^{2}\right)}{360 \pi} \cdot M+\frac{1}{96}\left(1+\left(l^{2}-1\right)\left(\cos \left(\varphi_{\mathrm{U}}\right)\right)^{2}\right)\right]
\end{aligned}
$$

Fig. 6a shows $I_{\mathrm{B}}=\Delta I_{\text {SYPWM }} /\left(\frac{T_{\mathrm{P}}}{2} \cdot \frac{U_{\mathrm{dc}}}{l L_{\mathrm{d}}}\right)$ with $L_{\mathrm{d}}=0.3 \mathrm{mH}$ and $L_{\mathrm{q}}=1.5 \mathrm{mH}$. The results for $\Delta I_{3 \mathrm{rdPWM}}$ do not differ significantly from those of $\Delta I_{\text {SYPWM }}$.

For discontinuous modulation methods (DPWM), different distributions of the freewheeling states are commonly used [5]. The reference functions for two of them are shown in fig. $5 \mathrm{c}$ and $5 \mathrm{~d}$. 
Because of a distinction of cases in the turn-on times for DPWM1 within sector I and the resulting length in the expression for the current harmonics, DPWM1 will not be further investigated within this paper. In the case of DPWM2 the relative turn-on times are

$$
\frac{t_{7, \mathrm{DPWM} 2}}{T_{\mathrm{p}} / 2}=0 \quad \frac{t_{8, \mathrm{DPWM} 2}}{T_{\mathrm{p}} / 2}=1-\frac{t_{1}}{T_{\mathrm{p}} / 2}-\frac{t_{2}}{T_{\mathrm{p}} / 2}
$$

These lead to the result for the current harmonics for DPWM2:

$$
\begin{aligned}
& \Delta I_{\mathrm{DPWM}}^{2}=\left(\frac{T_{\mathrm{P}}}{2}\right)^{2}\left(\frac{U_{\mathrm{dc}}}{l L_{\mathrm{d}}}\right)^{2} M^{2} . \\
& {\left[\left(\frac{3\left(1+\left(l^{2}-1\right)\left(\cos \left(\varphi_{\mathrm{U}}\right)\right)^{2}\right)(3 \sqrt{3}+8 \pi)}{1024 \pi}-\frac{27\left(l^{2}-1\right) \cos \left(\varphi_{\mathrm{U}}\right) \sin \left(\varphi_{\mathrm{U}}\right)}{1024 \pi}\right) M^{2}-\right.} \\
&\left(\frac{\sqrt{3}\left(183\left(\cos \left(\varphi_{\mathrm{U}}\right)\right)^{2}\left(l^{2}-1\right)+179-4 l^{2}\right)}{1440 \pi}+\frac{45\left(l^{2}-1\right) \cos \left(\varphi_{\mathrm{U}}\right) \sin \left(\varphi_{\mathrm{U}}\right)}{1440 \pi}\right) M+ \\
&\left.\frac{1}{24}\left(1+\left(l^{2}-1\right)\left(\cos \left(\varphi_{\mathrm{U}}\right)\right)^{2}\right)\right]
\end{aligned}
$$

The corresponding surface can be seen in fig. $6 \mathrm{~b}$.

Fig. 7 shows the results of the calculated current harmonics for SPWM, SYPWM and DPWM2 for stator voltage angles $\varphi_{\mathrm{U}}=\pi$ and $\varphi_{\mathrm{U}}=\frac{\pi}{2}$. Since the pulse period $T_{\mathrm{p}}$ for each of the three modulation methods has been considered as constant, for discontinuous modulation there are less switching operations within one pulse period. At an angle $\varphi_{\mathrm{U}}=\pi$ this causes higher current harmonics for DPWM, especially at lower modulation depths. With higher modulation depths, the difference becomes smaller.

As can be seen in fig. $7 \mathrm{~b}$, the impact of less switching operations within one pulse period decreases as $\varphi_{U}$ changes towards the direction of the d-axis. For $\varphi_{U}=\frac{\pi}{2}$ the resulting current harmonics for all three investigated modulation methods are almost equal.

\section{Correlation to the operating point of the IPMSM}

The inner voltage components of the machine are dependent on the operation point of the machine. They correspond to the speed of the machine and the current reference values $i_{\mathrm{d}}^{*}=i_{\mathrm{d}, 0}$ and $i_{\mathrm{q}}^{*}=i_{\mathrm{q}, 0}$ of the torque control. For IPMSM, $i_{\mathrm{d}}^{*}$ and $i_{\mathrm{q}}^{*}$ can be determined with look-up-tables of the trajectory of maximum torque per ampere (MTPA) for lower speeds and along the voltage constraint for the fieldweakening area [6]. By use of eq. (18) the modulation depth $M$ and the voltage phase angle $\varphi_{\mathrm{U}}$ can be determined for every operation point.

\section{Simulation results}

Simulations have been made in Matlab/Simulink for a high-saliency IPMSM with constant inductances $L_{\mathrm{d}}$ and $L_{\mathrm{q}}$. Simulation parameters are shown in table I.

\begin{tabular}{|cc|cc|}
\hline DC link $U_{\mathrm{dc}}$ & $300 \mathrm{~V}$ & Flux linkage $\Psi_{\mathrm{PM}}$ & $0.065 \mathrm{Vs}$ \\
\hline Pulse period $T_{\mathrm{p}}$ & $100 \mu \mathrm{s}$ & Inductance $L_{\mathrm{d}}$ & $0.35 \mathrm{mH}$ \\
\hline pole pairs $p$ & 3 & Inductance $L_{\mathrm{q}}$ & $1.5 \mathrm{mH}$ \\
\hline
\end{tabular}

Table I: Simulation parameters

The desired torque value of $T=80 \mathrm{Nm}$ has been generated with the current reference values shown in fig. 8a. They correspond to the MTPA-curve for lower speeds, whereas at higher speed the current reference values have been chosen for operation at maximum modulation depth. The corresponding modulation depth $M$ and voltage angle $\varphi_{\mathrm{U}}$ can be seen in fig. 8b. During MTPA-operation $\varphi_{\mathrm{U}}$ stays constant, while $M$ increases proportional to the speed of the machine. As the maximum modulation depth is reached, $M$ stays constant, while $\varphi_{\mathrm{U}}$ increases towards values $>\pi$. 


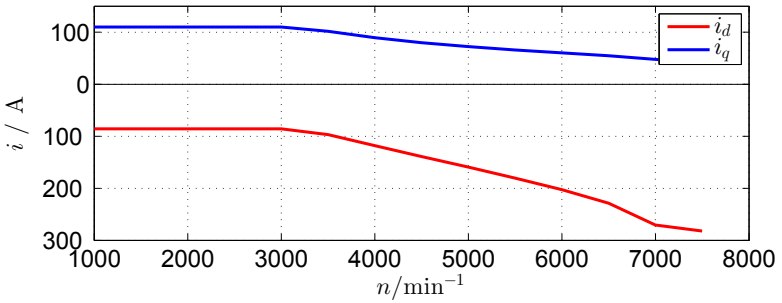

(a)

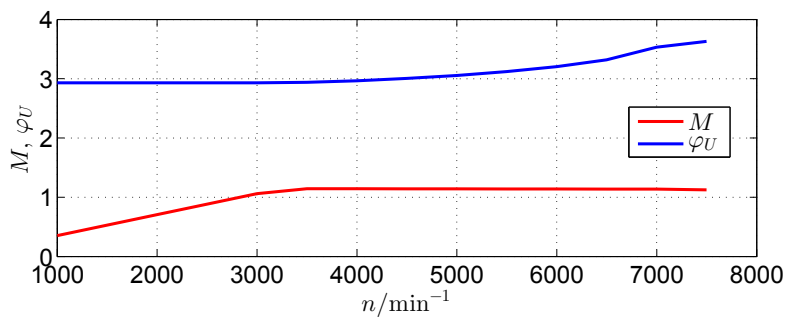

(b)

Figure 8: Current components (a) and modulation depth and voltage angle (b) for a torque of $T=80 \mathrm{Nm}$.

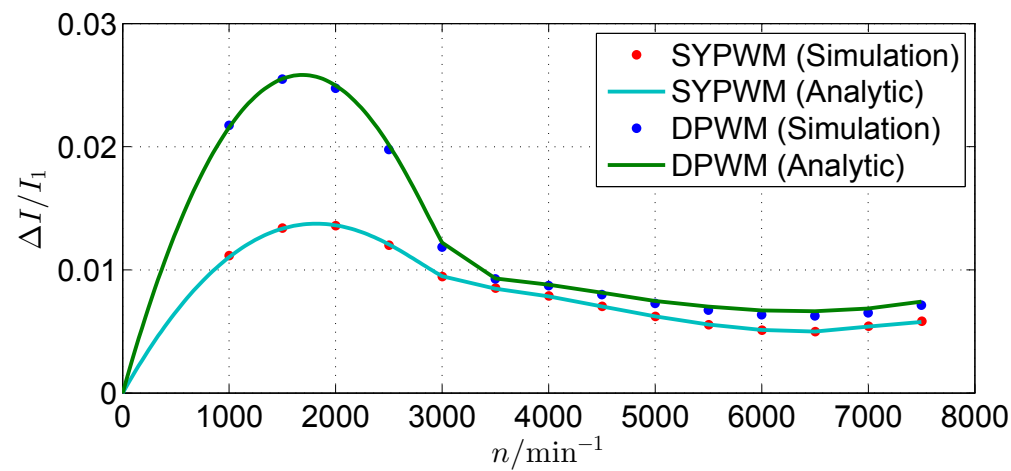

Figure 9: Simulation and analytic results of $\Delta I / I_{1}$ for two different modulation methods.

Fig. 9 shows the results of the simulation for SYPWM and DPWM2. The effect of $L_{\mathrm{d}} \neq L_{\mathrm{q}}$ on the current harmonics can clearly be seen: In the field weakening area, with constant modulation depth $M$, the current harmonics do not stay constant. They vary with increasing speed because of the shift of $\varphi_{U}$. The rms values of the current harmonics have also been calculated with the derived formulas. As can be seen, the analytic values comply very well to the harmonics in simulation. Therefore, the derived formulas for the current harmonics apply to machines assuming constant inductance values $L_{\mathrm{d}}$ and $L_{\mathrm{q}}$.

\section{Experimental results}

Measurements of current harmonics have been made with an IPMSM with parameters similar to those in tab. I. The current distortions have been measured with a Norma D6000. During measurements, the angle of the voltage space vector has been constant, while the modulation depth has been varied by increasing the speed of the machine. With current components $i_{\mathrm{d}}=-100 \mathrm{~A}, i_{\mathrm{q}}=50 \mathrm{~A}$ for $\varphi_{\mathrm{U}} \approx \pi$ and $i_{\mathrm{d}}=-100 \mathrm{~A}, i_{\mathrm{q}}=10 \mathrm{~A}$ for $\varphi_{\mathrm{U}} \approx \frac{\pi}{2}$ the desired angles of the voltage space vector were realized. The results for the total harmonic distortion, which is being defined as

$$
T H D=\frac{\sqrt{I_{\mathrm{rms}}^{2}-I_{1}^{2}}}{I_{1}}
$$

can be seen in fig. 10, together with a 4th order polynomial interpolation through the setpoints. The dashed lines in fig. 10 show the results of eq. (35) and (37).

There is a significant offset between the analytic and the experimental results in the THD-value for both modulation methods. This is due to the following cases:

- The IPMSM cannot be driven in an open-loop control. Therefore closed-loop current control is being used, which itself causes additional harmonics in the phase currents.

- Saturation effects of the inductances might have a significant impact on the current distortion. Although the evaluation of eq. (35) and (37) has been done with inductance values which have been derived from parameter measurements of the machine, their values still might differ from those that are essential for the current harmonics. 


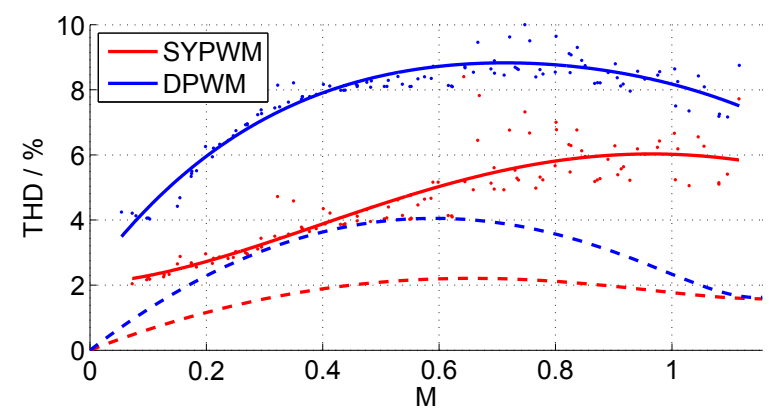

(a)

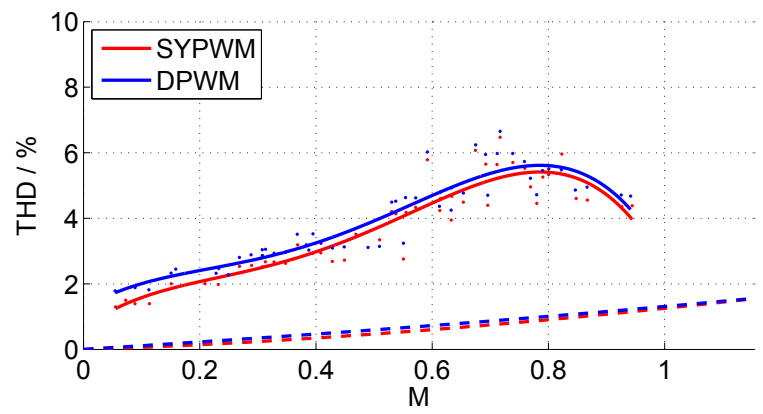

(b)

Figure 10: Measured harmonic distortion for use of different modulation methods. (a): $\varphi_{U} \approx \pi$. (b): $\varphi_{U} \approx \frac{\pi}{2}$.

- During measurements, the voltage vector angle $\varphi_{U}$ might slightly differ from the desired one, which also influences the current distortion. This could be the reason for the measured THD values decreasing at higher modulation depths at $\varphi_{U} \approx \frac{\pi}{2}$, although the calculated estimated values are monotonously increasing with the modulation depth.

A closer investigation of these issues will be carried out in future work.

However, the shape of the measured THD values with constant $\varphi_{U}$ and varying modulation depth and especially the relative comparison between the two illustrated modulation methods clearly corresponds to the shape of the calculated values for the rms value of the harmonic currents. At $\varphi_{U} \approx \pi$, the difference between the distortion with use of SYPWM and DPWM is significantly large, especially at modulation depths $M<0.8$. As the modulation depth becomes larger, the difference decreases. For $\varphi_{\mathrm{U}} \approx \frac{\pi}{2}$, the measured current distortions for both SYPWM and DPWM are almost equal, although the number of switching operations within one pulse period at DPWM is reduced by $\frac{1}{3}$ compared to SYPWM.

\section{Conclusion and future work}

Analysation of the current distortion in interior permanent magnet synchronous machines for different modulation methods has been presented in this paper. Analytic equations for estimation of harmonics have been derived and verified by numerical simulation. The results might be used to optimize the drive system by choosing the optimal modulation method and using the ideal switching frequency with respect to the operation point in order to minimize the total losses.

Measurements on a machine test bench have shown that the qualitative results for the current distortions can be used for a comparison of different modulation methods regarding the resulting current harmonics. The absolute values do not comply to the experimental results. The reasons for these discrepancies will be subject to further investigations.

\section{References}

[1] Van der Broeck H.W. and Skudelny H.-C.: Analytical Analysis of the Harmonic Effects of PWM AC Drive, IEEE Transactions on power electronics, vol 3, no 2, pp 216-223, Apr 1988

[2] Kolar J. W. , Ertl H. and Zach F. C.: Influence of the Modulation Method on the Conduction and Switching Losses of a PWM Converter System, IEEE Transactions on industry applications vol 27, no 6, pp 1063-1075, Nov/Dec 1991

[3] Bhavsar T. and Narayanan G.: Harmonic Analysis of Advanced Bus-Clamping PWM Techniques, IEEE Transactions on power electronics, vol 24, no 10, pp 2347 - 2352, Oct 2009

[4] Aguirre M., Madian P, Poza J. Aranburu A. and Nieva T.: Analysis and Comparison of PWM Modulation Methods in VSI-Fed PMSM Drive Systems, 2012 XXth International Conference on Electrical Machines (ICEM), pp 851-857, Sep 2012

[5] Hava A.M., Kerkman R.J.and Lipo, T.A.: A high-performance generalized discontinuous PWM algorithm, IEEE Transactions on Industry Applications, vol 34, no 5, pp 1059-1071, Sep/Oct 1998

[6] Morimoto S., Sanada M. and Takeda Y.: Wide-speed operation of interior permanent magnet synchronous motors with high-performance current regulator, IEEE Transactions on industry applications, vol 30, no 4, pp 920-926, Jul/Aug 1994 